\title{
Optimized Electrification of Subsea Oil \& Gas Infrastructures Based in Genetic Algorithm
}

\author{
Tiago A. Antunes ${ }^{1}$, Rui Castro ${ }^{2}$, P. J. Santos ${ }^{3}$, A. J. Pires ${ }^{4}$ and Matthias Foehr ${ }^{5}$ \\ ${ }^{1}$ Siemens SA, Energy Management Transmission Solutions, Lisbon, Portugal, \\ tiago.antunes@siemens.com; ${ }^{2}$ INESC-ID/IST University of Lisbon, Portugal, \\ rcastro@tecnico.ulisboa.pt; ${ }^{3}$ INESC Coimbra; EST Setúbal Department of Electrical \\ Engineering, Setúbal, Portugal, paulo.santos@estsetubal.ips.pt; ${ }^{4}$ CTS/UNINOVA; EST \\ Setúbal/IPS Department of Electrical Engineering, Setúbal, Portugal, \\ armando.pires@estsetubal.ips.pt; ${ }^{5}$ Siemens AG, Energy Management Transmission Solutions, \\ Erlangen, Germany, matthias.foehr@siemens.com;
}

\begin{abstract}
Offshore field development relies on multiple optimization techniques targeting a feasible and cost-effective production solution yet are focused on the field itself. While so, advancements in offshore engineering bring increasingly complex subsea infrastructures to depths in the excess of 3,500 meters. Many offshore production topsides which currently rely on costly and harmful onboard thermal-based power generation are turning to high voltage power-from-shore electrification solutions to cope with the challenges being brought by subsea infrastructures. An optimal electrification of these subsea templates is a challenge on its own as the seafloor morphology and well distribution is far from consistent. This paper presents a combined k-means and genetic-algorithm optimization to assess how the combined deployment of high voltage umbilical, wellheads and subsea substations can be optimized for the lowest cost possible. Results show a significant improvement in optimization of the total umbilical length as well as the substation positioning on the seabed.
\end{abstract}

Keywords: subsea, substations, oil \& gas, optimization, genetic algorithm

\section{Introduction}

The International Energy Agency (IEA) stresses that projects in the main areas such as the Gulf of Mexico and the North Sea, were once characterized by a break-even ratio of 60-80USD/barrel. Equivalent projects are made viable and as robust as breakeven oil prices of 25-40 USD/barrel [1]. This can only be achieved via proper simplification, modularization and downsizing; Electrification is at the core [2][3].

Offshore power production is based in thermal combustion systems whether this is accomplished via diesel or gas and that can reach several dozen megawatts [4][5]. Modularization and optimization mean that owners can increase the complexity of the templates, reduce the size of the topside and increase the overall efficiency and resilience. Furthermore, unmanned operations, expected to dramatically reduced Operational Expenditure (OpEx), will require a reliable power supply with a large maintenance window, possible by means of power-from-shore (PFS) solutions [6]. 
In subsea facilities commonly ranging up to $100 \mathrm{MW}$ [7] the matter is addressed on a point-to-point or direct in-place analysis of the most suitable electrical infrastructure and not on the optimization of the actual electrical grid on the seafloor. The authors have reviewed field development cases [8][9], yet those references are focused on the subsea production setup and not on the actual electrification.

The authors were able to review several case studies where satisfactory results were obtained by means of GA [10][11][12] - which sustains the suitability of the approach. The same algorithm was also used in other examples, such as in the design of S/S's earthing grids [13], switchgear internal design [14] or in life-cycle assessment of electrical S/S [15]. References show that simulated annealing can also be applied for this sort of optimization, often times combined with GA [16][17].

The rest of the paper is organized as follows. On the second chapter, a connection is established between this research and the resilient systems topic. The third chapter provides an overview of the main industry challenges, which shall serve, on the forth chapter, to support the model design. The results and conclusions are presented on the fifth and sixth chapter, respectively.

\section{Relationship to Industrial and Service Systems}

Although PFS on itself is not an innovation, and field development is a consolidated environment, no references to support the use of Genetic Algorithms (GA) or others for optimized subsea electrification were found. However, the application of GA on the optimization of power systems, more specifically, in the substation $(\mathrm{S} / \mathrm{S})$ expansion planning (SEP) is widespread and quite common. Companies like SIEMENS want to drive large-scale deployment of PFS solutions as an alternative to onboard thermal allowing utilities to take full advantage of the massive renewable capacity available.

Having so, and whilst the authors assess new ways to power Oil \& Gas (O\&G) infrastructures from shore, this paper presents their novelty approach towards bringing a much-needed solution for the cost optimization of the subsea electrical setup. It is designed by means of a Python-based mixed Kernel-Means (KM) and GA, considering simultaneously the layout of the field and the electrification concept to get the most cost-effective solution.

\section{Industry Challenges}

- An analysis of over one hundred offshore sites showed electrification costs ranging between 0.3-0.5 MUSD $/ \mathrm{kboed}^{1}$ despite the variations on the resource or water depth. In the case of subsea infrastructures, the ratio was 0.5 ;

- Subsea production facilities are characterized by largely dispersed and smallsized equipment. An analysis of all the possibilities can become timeconsuming and the GA does not require the evaluation of all possibilities [18];

\footnotetext{
${ }^{1}$ One thousand barrels of oil equivalent per day
} 
- Industry leaders are working towards subsea electrical power standardization, which supports the claim that robust subsea electrical systems are both new and indeed needed at a fast pace [19];

On one hand, unsupervised methods such as KM clustering bring a straightforward method to analyze the positioning of the wells without initial candidate locations [17]. As it does not require initial mapping it serves as a solid method to suggest individual candidates on the GA application [11]. On the other hand, GA are known for providing high quality and performance results, especially for a high number of possibilities [20]. In addition, they show enough flexibility to accommodate several restrictive measures - such as the number of power outlets allowed on a $\mathrm{S} / \mathrm{S}$ and the maximum cable distance.

\section{Proposed Method}

\subsection{Initial Assumptions}

An extensive analysis of the offshore portfolio was made. Wellheads are distributed along a squared area, representing field section. The number of wells is based on current projects. Focus was given to the evaluation of the best possible electrification for any given field development considering the following portfolio restrictions:

- Electrical distribution is made typically using voltages ranging between 3.3 and $36 \mathrm{kV}$ [19][7] and oil-filled, pressure-balanced and coated electrical distribution units (EDU or SDU) are used to power the subsea systems. These units rely on templates pre-assembled on surface [21];

- $\quad$ Electrical distribution manifolds (EDM) - a subsea substation, can also be used to provide power to multiple locations at the same field whilst the number of connectors is kept to a minimum to ensure overall system resilience [21];

- One power transformer is planned on each $\mathrm{S} / \mathrm{S}$ and the technology used, high voltage alternated current (HVAC) or direct current (HVDC), is not compared as it depends on the distance to shore; And this optimization serves for both;

- The number of power distribution outlets of the EDU is estimated between 3 and 6 at a given voltage level whereas subsea wellheads are assumed to be rated the same [3];

- The number of S/S is estimated based on the quantity of wellheads, as the cost of the PDU is largely higher than the HV umbilical an estimation based solely in the number of connections to do was used [21];

- The initial positioning of the $\mathrm{S} / \mathrm{S}$ is done via the K-means clustering method whereas the GA populates multiple candidate substation positioning. The size of the solution array is constant which means that all subsea templates are powered by one and only one of the subsea $\mathrm{S} / \mathrm{S}$;

- The solutions (individuals) share traits ( $\mathrm{S} / \mathrm{S}$ connections) between each other and the GA application is able, not only to foster the better combination of those traits, but also, to short-list the better candidate solutions [22]. 
A joint approach on both hydraulic and electrical power subsea distribution was not made although it may lead to further improvements. The geography of the seabed and fields are outside the focus of this paper. The objective is an universal canvas.

\subsection{Process Flow}

As said, KM is used as an unsupervised learning method for clustering the field data. This algorithm provides each individual with a candidate position and the distance to each wellhead to which respects to. Please refer to Fig. 1. for a process flow example. Subsea wells are identified in blue whereas the S/S are identified in green. The links are mapped accordingly. This is the baseline for the GA, representing one individual from the sense of the $\mathrm{S} / \mathrm{S}$ location.
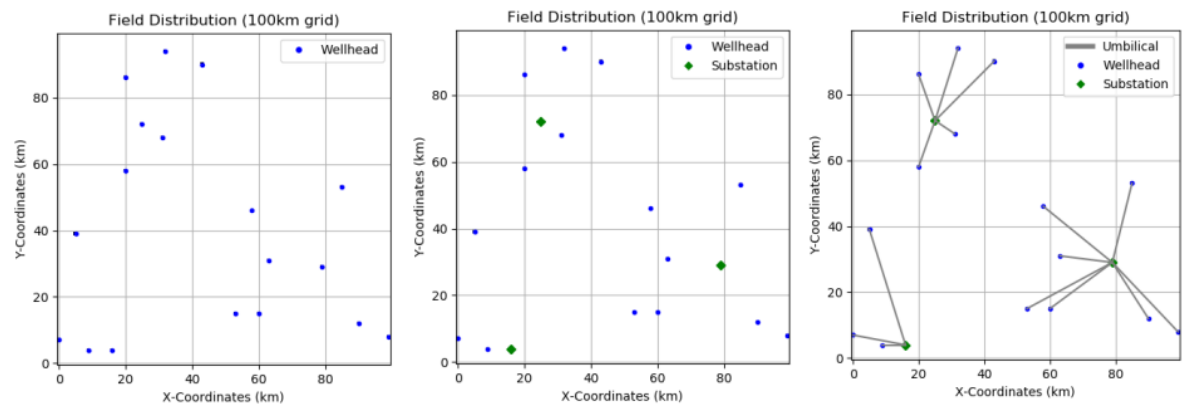

Fig. 1. Deployment of wellheads, substations and umbilical (algorithm process)

For any well and subsea S/S set, the objective is to minimize the total cost of the electrification solution (cumulative cost of the subsea cabling for each well-substation link). The cost objective function (1) to be minimized is presented next, where $\mathrm{L}$ represents each cable distance, $\mathrm{C}$ and $\mathrm{C}_{\mathrm{KM}}$ the total and unitary cost, respectively:

$$
\mathrm{C}=\sum \mathrm{L}_{\mathrm{CABLE}-\mathrm{N}} * \mathrm{C}_{\mathrm{KM}}
$$

The connections are represented via a single-digit code which states the reference of the respective $\mathrm{S} / \mathrm{S}$. That allows for a direct and natural encoding. The same node (position) cannot be completed by neither none nor more than one $\mathrm{S} / \mathrm{S}$ - an example is shown on Fig. 2. Script-wise, each individual is made of an integer array with a length equal to the number of load centres (wells).

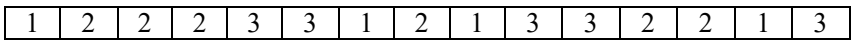

Fig. 2. Example of an individual as used on the GA

The distance is calculated as the shortest straight line between the two-points ( $\mathrm{S} / \mathrm{S}$ and well). Such calculation outputs a similarly-structured array. The decision variable is the total cost for an individual (composed of the cumulative cost of each wellhead/substation tuple). Typically, for this analysis, the restrictions are the spatial 
representation of the wells, S/S and connection links. All individuals are deemed acceptable and the tuples are selected within the number of wells and S/S proposed.

\subsection{Characterization of the GA Population}

The population size was set so that a balance between both the number of S/S and templates to power is reached. Sensibility tests were made to assess the appropriate setup. The best compromise was found sizing a population equal to the number of wellheads (links) with a medium crossover rate and a very low mutation rate. The iteration rate was kept high during most cases, although fitness is achieved quite early. The population size is the most critical variable although the mutation rate must be kept to a minimum (up to $10 \%$ ) given the large population size. [7].

Table 1. Constrains of the Genetic Algorithm

\begin{tabular}{llll}
\hline Rate designation & Variable & Value & Units \\
\hline Population (individuals) & ind_rate & 1 & {$[-]$} \\
Iterations of the GA & ite_rate & 200 & {$[-]$} \\
Top fitness rate & - & 1 & {$[-]$} \\
Crossover rate & cross-rate & 40 & $\%$ \\
Mutation rate & mut_rate & 10 & $\%$ \\
\hline
\end{tabular}

\subsection{Ranking, Crossover and Mutation}

The total cost per each individual is calculated by means of the sum of the length of all links combined and the unitary cost. Fig. 3 presents an example of the elite selection by addressing the total cost (per individual) array of the population.

\begin{tabular}{|l|l|l|l|l|l|l|l|l|l|l|l|l|l|l|}
\hline 10 & 22 & 35 & 12 & 14 & 18 & 19 & 20 & 12 & 23 & 25 & 17 & 15 & 20 & 22 \\
\hline
\end{tabular}

Fig. 3. Example of a cost array as used on the GA

A portion of the remaining population is selected for cross-over. An even number of individuals are selected, and a proper parent-children pair can be selected. The cross-over is done using the sexual reproduction principal with two individuals, done via cross-over pointing, where the two new sub-set strings are swapped and will form the children individuals. An example is presented in Fig. 4.

\begin{tabular}{|l|l|l|l|l|l|l|l|l|l|l|l|l|l|l|l|}
\hline Parent A & 1 & 2 & 2 & 2 & 3 & 3 & 1 & 2 & 1 & 3 & 3 & 2 & 2 & 1 & 3 \\
\hline Parent B & 1 & 3 & 1 & 1 & 2 & 2 & 3 & 3 & 2 & 2 & 2 & 1 & 3 & 3 & 2 \\
\hline Child A & 1 & 2 & 2 & 2 & 3 & 3 & 3 & 3 & 1 & 3 & 3 & 2 & 2 & 1 & 3 \\
\hline Child B & 1 & 3 & 1 & 1 & 2 & 2 & 1 & 2 & 2 & 2 & 2 & 1 & 3 & 3 & 2 \\
\hline
\end{tabular}

Fig. 4. Example of a cross-over operation as used on the GA 
The parents are randomly selected, and each tuple provides one children. For that reason, the number of cross-over iterations matches the number of parents (population size remains unchanged). There is no preference on the parents and the two new individuals from each round proceed for the mutation pool.

The mutation is done to the pool of all individuals with an exception for the top fitness one, thus including the untouched individuals, as well as, the cross-over children. The low rate mutation is applied on a single random point of an individual. One link ( $\mathrm{S} / \mathrm{S}$ reference) is changed in that percentage of individuals. In order not only to keep accurate results and avoid a recalculation of the costs, but more importantly, to reanimate possible important solutions, the "mutated" individual receives a gene (link and distance tuple) from another individual of the pool, also randomly selected. The process is shown in Fig. 5.

\begin{tabular}{|l|l|l|l|l|l|l|l|l|l|l|l|l|l|l|l|}
\hline Original & 1 & 2 & 2 & 2 & 3 & 3 & 1 & 2 & 1 & 3 & 3 & 2 & 2 & 1 & 3 \\
\hline Mutated & 1 & 2 & 2 & 2 & 3 & 3 & 1 & 3 & 1 & 3 & 3 & 2 & 2 & 1 & 3 \\
\hline
\end{tabular}

\begin{tabular}{|l|l|l|l|l|l|l|l|l|l|l|l|l|l|l|l|} 
Donor & 1 & 3 & 1 & 1 & 2 & 2 & 3 & 3 & 2 & 2 & 2 & 1 & 3 & 3 & 2 \\
\hline
\end{tabular}

Fig. 5. Example of a mutation operation as used on the GA

The distance is already available, taken also from the equivalent position on the distances' array. This avoids the need to recalculate all distances of the current population and optimizes computation time. The standard deviation is used to assess the performance of the GA. This process is iterative and is repeated for a given number of times.

\section{Results}

\subsection{Initial Conditions}

The test conditions are presented in Table 2 .

Table 2. Key data of the Case Study

\begin{tabular}{lll}
\hline Constrain description & Value & Units \\
\hline Map resolution & 100 & $\mathrm{~km}$ \\
Number of substations & 3 & {$[-]$} \\
Number of wellheads & 15 & {$[-]$} \\
Number of iterations & 1,000 & {$[-]$} \\
Unitary umbilical cost & 5,000 & USD $/ \mathrm{km}$ \\
\hline
\end{tabular}

The mapping is reviewed against the subsea grids and $O \& G$ fields known. The cost for the umbilical is indicative and the number of $\mathrm{S} / \mathrm{S}$ is selected based on maximum number of connections of each unit (PDU), in this case 5 wellheads each. The optimized deployment is shown on Fig. 5. The grey line represents the HV umbilical laid between the wellheads and the respective $\mathrm{S} / \mathrm{S}$. The authors acknowledge that the 
solutions proposed are not perfect and additional measures should be taken to avoid dispersion of the $\mathrm{S} / \mathrm{S}$ along the field during GA execution.
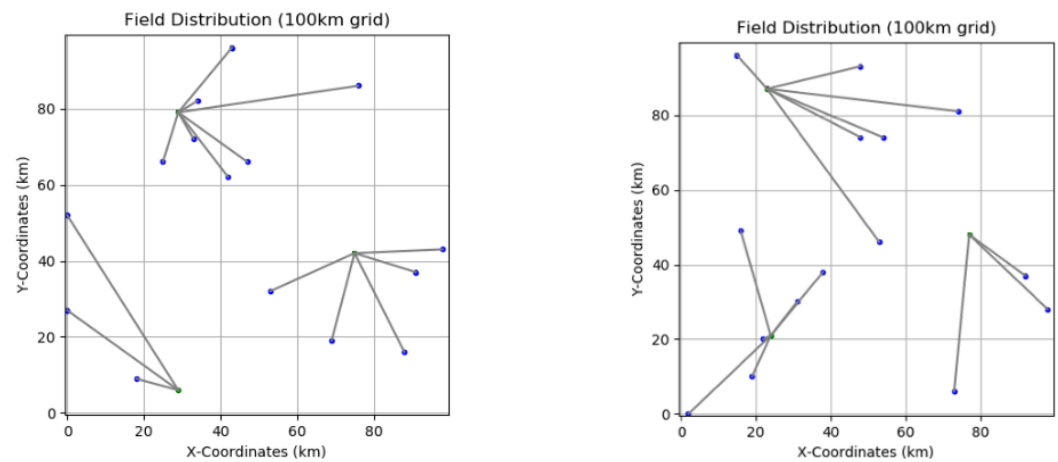

Fig. 6. Field distribution with substations deployed (Case studies)

\subsection{Cost Minimization}

The results of the total distance and cost for each iteration are presented in Figure 6.
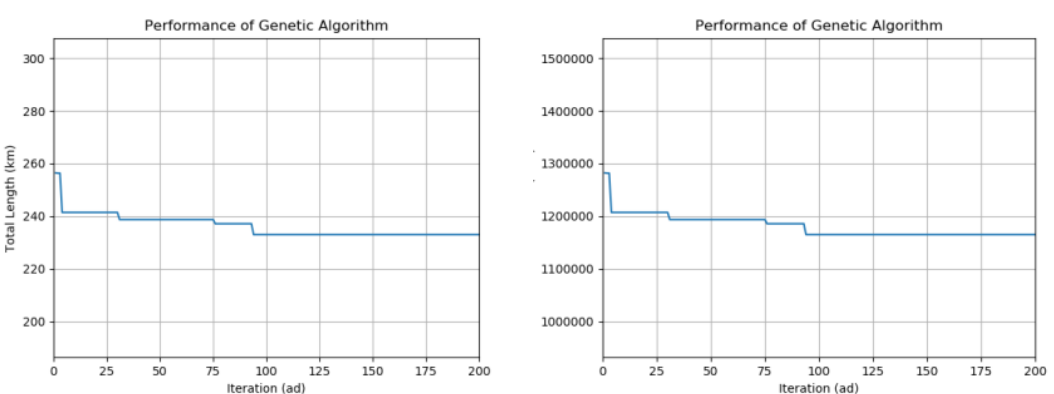

Fig. 7. $\{$ Cost, distance $\}$ per iteration

In the same manner, the standard deviation is presented in Figure 7. 

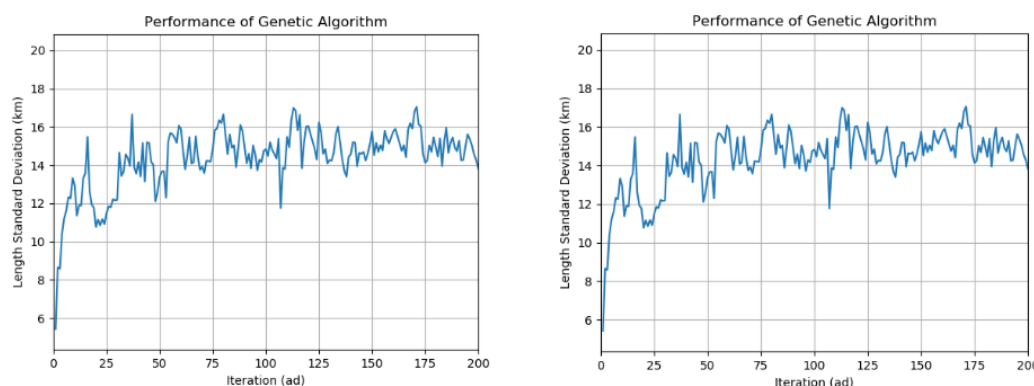

Fig. 8. \{Standard distance deviation, cost deviation $\}$ per iteration

There is a quick convergence towards the best cost position. However, as already commented, the higher rate of iterations was defined so that a sustained convergence of the overall population was possible. Results are summarized on Table 3.

Table 3. Final results

\begin{tabular}{lll}
\hline Variable identification & Value & Units \\
\hline Total of Iterations & 1,000 & - \\
Initial Length & 258 & $\mathrm{~km}$ \\
Final Length & 235 & $\mathrm{~km}$ \\
Initial Cost & 1,280 & MUSD \\
Final Cost & 1,170 & MUSD \\
\hline
\end{tabular}

In some scenarios, the same cost was found for two simultaneous positions. Yet, these are a minor occurrence on the overall spectrum of scenarios addressed. The routine is, however, able to identify the individuals which match this best fitness performance.

In fact, using a KM before the GA implementation dramatically reduces the computation time for the optimization as the centroids are calculated (via the Euclidean distance) close to their final positions in the majority of cases. Increasing the threshold was used to accommodate the lack of convergence, in multiple cases, of the standard deviation. Efforts were made to adjust the cross-over and mutation ratios to improve such convergence.

An increase on the mutation rate is known to allow for an optimization of the computation of the solution which supports the claim that associative optimization problems can be served with better results by using mutation rates higher than crossover rates. The increased cross-over ration introduces a very high disturbance on the standard deviation, which should be avoided. On the other hand, the increase mutation factor causes the abrupt convergence of the least cost having a similar deviation conversion as of the initial case scenario. 


\section{Conclusions}

It was concluded that the paper presents an appropriate solution for an optimized deployment of a subsea infrastructure by means of a KM and GA-based routine. A satisfactory optimization of the total cost was achieved, and an appropriate narrowing of the individual's fitness was also accomplished. The authors also acknowledge that there is enough room to innovate as multiple deployment restrictions have to be accounted for in such models.

The authors trust that a successful tool will certainly include additional optimization tiers (still using the GA or other suitable algorithm) so that topics like the substation positioning vs the HV umbilical, avoidance of unsuitable paths for the links or maximum tie-in length topics are addressed.

\section{References}

1. IEA (2017) Offshore Energy Outlook

2. Rajashekara K, Krishnamoorthy H (2018) Power electronics for subsea systems: Challenges and opportunities. In: Proceedings of the International Conference on Power Electronics and Drive Systems

3. Rajashekara K, Krishnamoorthy HS, Naik BS (2017) Electrification of Subsea Systems : Requirements and Challenges in Power Distribution and Conversion. Cpss Trans Power Electron Appl 2:. https://doi.org/10.24295/CPSSTPEA.2017.00024

4. Shi G, Peng S, Cai X, et al (2012) Grid integration of offshore wind farms and offshore oil/gas platforms. In: Conference Proceedings - 2012 IEEE 7th International Power Electronics and Motion Control Conference - ECCE Asia, IPEMC 2012. pp $1301-1305$

5. Hamdan HA, Kinsella B (2017) Using a VSC Based HVDC Application to Energize Offshore Platforms from Onshore - A Life-cycle Economic Appraisal. In: Energy Procedia. pp 3101-3111

6. Yamamoto M, Almeida CFM, Angelico BA, et al (2014) Integrated subsea production system: An overview on energy distribution and remote control. In: 2014 IEEE Petroleum and Chemical Industry Conference - Brasil, PCIC Brasil 2014

7. Hazel T, Baerd HH, Legeay JJ, Bremnes JJ (2013) Taking power distribution under the sea: Design, manufacture, and assembly of a Subsea electrical distribution system. IEEE Ind Appl Mag. https://doi.org/10.1109/MIAS.2012.2215648

8. Wang Y, Gu J, Duan M, et al (2016) A new partition model for the optimization of subsea cluster manifolds based on the new definition of layout cost. Proc Inst Mech Eng Part M J Eng Marit Environ. https://doi.org/10.1177/1475090215590466

9. Wang Y, Duan M, Feng J, et al (2014) Modeling for the optimization of layout scenarios of cluster manifolds with pipeline end manifolds. Appl Ocean Res. https://doi.org/10.1016/j.apor.2014.02.006

10. Taroco CG, Carrano EG, Neto OM, et al (2012) A Faster Genetic Algorithm for Substation Location and Network Design of Power Distribution Systems. 2012 Ieee Congr Evol Comput 10-15

11. Vahedi S, Banejad M, Assili M (2015) Pseudo-dynamic substation expansion planning using hybrid heuristic and genetic algorithm. In: 2015 4th International Conference on Electric Power and Energy Conversion Systems (EPECS)

12. Gen M, Syarif A (2005) Hybrid genetic algorithm for multi-time period 
production/distribution planning. In: Computers and Industrial Engineering

13. Ghania SM (2016) Optimum design of grounding system inside high voltage substations for transient conditions. In: ICHVE 2016 - 2016 IEEE International Conference on High Voltage Engineering and Application

14. Hinow M (2008) Genetic algorithm based methodology for optimisation of innovative switchgear design. In: Conference Record of IEEE International Symposium on Electrical Insulation

15. Hinow M, Mevissen M (2011) Substation maintenance strategy adaptation for lifecycle cost reduction using genetic algorithm. IEEE Trans Power Deliv. https://doi.org/10.1109/TPWRD.2010.2065247

16. Adler D (1993) Genetic algorithms and simulated annealing: A marriage proposal. In: IEEE International Conference on Neural Networks - Conference Proceedings

17. Hongbo S, Shuxia LI, Degang G, Peng LOU (2006) Genetic simulated annealing algorithm-based assembly sequence planning. In: International technology and innovation conference, ITIC 2006.

18. Rexhepi A, Maxhuni A, Dika A (2013) Analysis of the impact of parameters values on the Genetic Algorithm for TSP. IJCSI Int Conf Comput Sci Issues

19. Asa S, Noel C, Alford M, Lanier S (2013) Subsea Electrical Power Standardization. In: PCIC Europe 2013. IEEE, pp 1-7

20. Rothlauf F (2006) Representations for Genetic and Evolutionary Algorithms, 2nd Editio. New York

21. Bai Y, Bai Q (2010) Subsea Engineering Handbook

22. Rothlauf F (2012) Representations for evolutionary algorithms 